\title{
Characterization of residual stresses and accumulated plastic strain induced by shot peening through simulation of instrumented indentation
}

\author{
Yu Gang $\mathrm{Li}^{1, \mathrm{a}^{*}}$, Pascale Kanouté ${ }^{1,2, \mathrm{~b}}$ and Manuel François ${ }^{1, \mathrm{c}^{*}}$ \\ ${ }^{1}$ Université de Technologie de Troyes (UTT), ICD-LASMIS, UMR CNRS 6281, 12, rue Marie Curie - \\ CS 42060, 10010 Troyes Cedex, France \\ ${ }^{2}$ The French Aerospace Lab (ONERA), DMSM/MCE, 29 avenue de la Division Leclerc-BP 72, \\ F-92322 Chatillon Cedex, France \\ ayugang.li@utt.fr, ${ }^{\mathrm{b}}$ Pascale.Kanoute@onera.fr, ${ }^{\mathrm{c}}$ manuel.francois@utt.fr
}

\begin{abstract}
Keywords: Finite element simulation, shot peening, residual stress, accumulated plastic strain,
\end{abstract} instrumented indentation

\begin{abstract}
In this study, a method based on finite element (FE) simulation was proposed for characterizing simultaneously residual stresses (RS) and accumulated plastic strain (PP) induced by shot peening process. Through a series of simulations of instrumented indentation, contour plots of several parameters, as maximum load, contact hardness and contact stiffness, were computed. By superposing them pair-wisely, RS and PP mixed together could be characterized simultaneously. In order to verify the method, several simulations with different combinations of RS and PP were performed. Results showed that the method is promising but remains to be tested on experimental acquisitions.
\end{abstract}

\section{Introduction}

Since nearly all of the mechanical processes, such as rolling, cutting, shot peening, simultaneously induce residual stresses (RS) and accumulated plastic strain (PP) into components, it would be interesting to develop a method to characterize quantitatively these two parameters. Indeed, mechanical methods such as hole drilling or layer removal only characterize RS but not PP. Diffraction methods, on the opposite can give RS through peak positions and, semi-quantitatively, can estimate PP through peak widths. For depth profiles, laboratory X-ray Diffraction (XRD) requires time consuming layer removal through electro-polishing. Instrumented indentation test (IIT), also known as depth-sensing indentation, allows the application of a specified force or displacement which could be controlled and measured simultaneously and continuously over a complete loading cycle. Many material properties, such as Young's modulus, hardness, yield stress, fracture toughness and so on, can be derived from this test. With instrumented indentation, it should be possible to obtain a RS and PP profile on a cross section of the specimen, even for complex gradients, complex specimen geometry, multilayered materials, amorphous materials. Recently, several methods and theoretical models were proposed [1-5]. However, some of these methods are only limited to the elastic domain $[2,4]$. Others models are suitable for the elastic-plastic domain, however the application to actual experiments is not very explicit [1]. Besides, the method allowing characterizing PP and RS at the same time is not well established as well. In this study, a strategy based on FE simulations of IIT, providing that the constitutive behavior of the material is known, is proposed for characterizing RS and PP induced by shot peening process. This strategy has the potential to be used with real experimental data.

\section{Theoretical Analysis}

The indentation tests performed in this study follow the standard ASTM E 2546-07. Considering a conical indenter equivalent to a Berkovich indenter as shown in Fig. 1(a), for which the semi apex angle $\alpha$ is equal to $70.3^{\circ}$, the evolution of the indentation load $(P)$ regarding the penetration depth $(h)$ (called $P$ - $h$ curve) is shown in Fig. 1(b). In a typical instrumented indentation process, contact stiffness $(S)$, contact hardness $(H)$ and projected contact area $(A)$ are respectively defined as: 


$$
\begin{aligned}
& S=\left.\frac{d P}{d h}\right|_{h \max }=2 E^{*} a \\
& H=\frac{P_{\max }}{A} \\
& A=\pi a^{2}
\end{aligned}
$$

where $a$ is the projected contact radius, $d p /\left.d h\right|_{h \max }$ is the initial slope of the unloading curve as shown in Fig. 1(b), $P_{\max }$ is the maximum load and $E^{*}$ is the reduced modulus which is expressed as:

$$
\frac{1}{E^{*}}=\frac{\left(1-v^{2}\right)}{E}+\frac{\left(1-v^{\prime 2}\right)}{E^{\prime}}
$$

$E$ and $v$ are the Young's modulus and the Poisson's ratio of the tested material and $E$ ' and $v^{\prime}$ are the Young's modulus and the Poisson's ratio of the indenter, respectively.

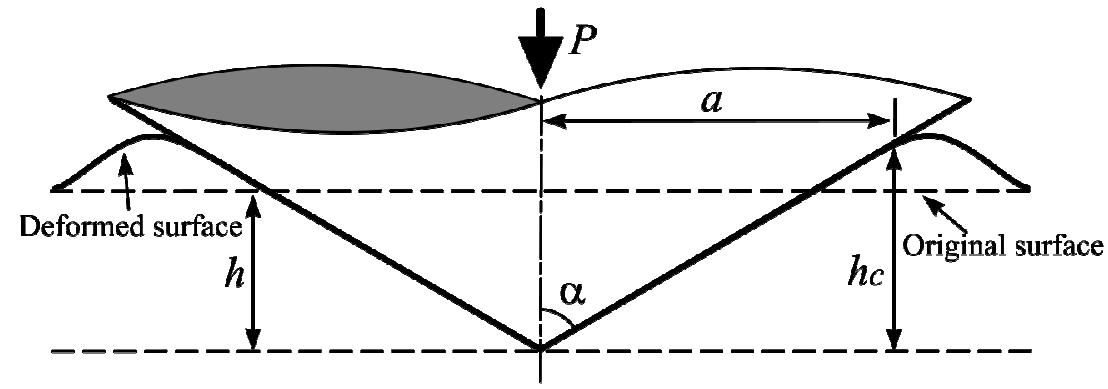

(a)

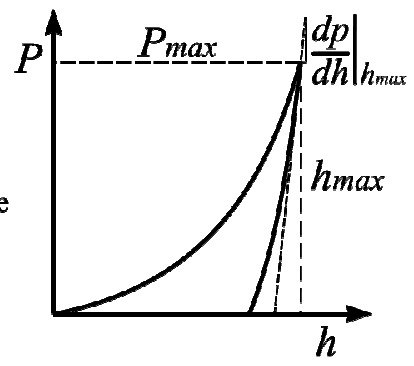

(b)

Fig. 1 Scheme of equivalent conical indenter and the $P$ - $h$ curve derived from it.

For a given penetration depth, both compressive residual stresses and work hardening (PP) induce an increase in $P_{\max }$ values as compared to the initial material $[2,4,6]$. In a previous work, it was shown that the introduction of RS or PP in the material leads to loading $P$ - $h$ curves almost superposed [6]. It is thus almost impossible to distinguish the two quantities from the analysis of the sole $P-h$ curves. However, it was also shown that, even when the curves are superposed, the amount of pile-up $\left(h_{c}-h\right)$ will vary which means that $A$ values will vary as well. A direct determination of $A$, for instance using the AFM technique, is inconvenient in practice. The idea proposed in the present paper is to use the contact stiffness $S$ which depends on $E^{*}$ and $A$. As shot-peening and similar processes do not, a priori, modify the Young's modulus of the specimen, it should not change $E^{*}$, and thus variations of $S$ are directly related to variations of $A$.

The $S$ value can be obtained easily and conveniently by fitting the initial slope of the unloading $P-h$ curve. The $P_{\max }$ value can be read directly on the $P$ - $h$ curve. These two independent quantities can be used, in principle, to determine the two quantities of interest: RS and PP. Alternatively, it can be seen that the hardness $H$ is also sensitive to the projected contact area $A$ and can be used (along with $P_{\max }$ ) to determine RS and PP. Assuming that the reduced modulus $E^{*}$ is unaffected by shot peening, it can be eliminated from Eq. 1 to Eq. 3:

$$
\frac{H}{H_{0}}=\frac{P_{\max }}{P_{\max 0}} \cdot\left(\frac{S_{0}}{S}\right)^{2}
$$

where index 0 stands for the untreated material. In practice, $H_{0}, P_{\max 0}$ and $S_{0}$ can be obtained through indentation tests far away from the peened surface. 


\section{Finite Element Simulation}

Considering the problem, a 2-D axisymmetric FE model is built. For this study, the commercial FE code ABAQUS/Standard (version 6.12-1) is used with implicit method. Considering a maximum indentation depth of $0.5 \mu \mathrm{m}$, the dimension of the whole model was set to $50 \mu \mathrm{m} \times 50 \mu \mathrm{m}$, which is assumed to be large enough to approximately represent a semi-infinite body. The whole model is considered as a representative volume element (RVE) loaded uniformly. Besides, considering the distributions of stress and strain fields in depth and in width due to the indentation test, the mesh in the zone beneath the indenter $(5 \mu \mathrm{m} \times 5 \mu \mathrm{m})$ was refined to guarantee the accuracy of the FE model. Moreover, simulations with different mesh size were performed to check the mesh sensitivity (results are not shown here). In order to represent the pile-up phenomenon precisely, a finest mesh size (down to $2.4 \mathrm{~nm}$ ) was used in the region where pile-up is expected to occur. Seeing the feature of indentation simulation, a four-node bilinear axisymmetric quadrilateral, reduced integration, hourglass control element (CAX4R) was used. The RS and PP are imposed independently by applying a uniform pressure, along the radial direction, on the external surface of the model. Plastic pre-strain is applied first until the desired level is reached. The model is then unloaded and re-loaded in the elastic range to simulate the residual stress field (assumed to be uniform at the RVE scale). The indenter is represented by an analytical rigid surface. The friction coefficient between the indenter and the material was taken equal to 0.15 . Quasi-static displacement controlled loading is applied to reach the desired indentation depth.

A Ludwik type isotropic hardening behavior is used, which is given as follows:

$$
\sigma=\sigma_{y}+K(P P)^{n}
$$

where $\sigma_{y}$ is the initial yield stress of the material, $P P$ is the accumulated plastic strain (work hardening), $K$ is the hardening coefficient and $n$ is the hardening exponent. This kind of relation between plastic true-stress and accumulated plastic true-strain is used because it gives the best fit for the uniaxial tensile test data in the strain range concerned by indentation tests. All the parameters in the constitutive model and their values are shown in Table 1 . They are based on experimental tensile tests performed on Ti40, a commercially pure $\mathrm{Ti}_{\alpha}$ alloy.

Table 1. Material properties considered in the numerical simulation.

\begin{tabular}{cccccc}
\hline Parameters & $\begin{array}{c}\text { Young's } \\
\text { modulus } E \\
{[\mathrm{GPa}]}\end{array}$ & $\begin{array}{c}\text { Poisson's } \\
\text { ratio } v \\
-\end{array}$ & $\begin{array}{c}\text { Yield } \\
\text { stress } \sigma_{y} \\
{[\mathrm{MPa}]}\end{array}$ & $\begin{array}{c}\text { Hardening } \\
\text { coefficient } K \\
{[\mathrm{MPa}]}\end{array}$ & $\begin{array}{c}\text { Hardening } \\
\text { exponent } n \\
-\end{array}$ \\
\hline Value & 122 & 0.33 & 800 & 340 & 0.1 \\
\hline
\end{tabular}

\section{Results and Discussion}

The discretization scheme adopted in the FE model was checked in the elastic range through a comparison with the analytical solution proposed by Sneddon and modified by Hay et al. [7, 8]. A very good agreement was found between the two $P$ - $h$ curves.

Next, a series of simulations on the elastic-plastic material with different levels of RS and PP were carried out. The levels of PP are respectively $0 \%, 1 \%, 5 \%, 10 \%, 15 \%$ and $20 \%$. The levels of RS are $0 \mathrm{MPa},-200 \mathrm{MPa},-400 \mathrm{MPa},-600 \mathrm{MPa},-800 \mathrm{MPa}-1000 \mathrm{MPa}$ and $-\sigma_{y}$, where $\sigma_{y}$ is the yield stress reached for each imposed PP level and is calculated from Eq. 6. Indentation simulation is performed for each combination of RS and PP. The normalized value of $P_{\max }, S$ and $H$ obtained from all the simulations vs RS and PP are shown in Fig. 2. 

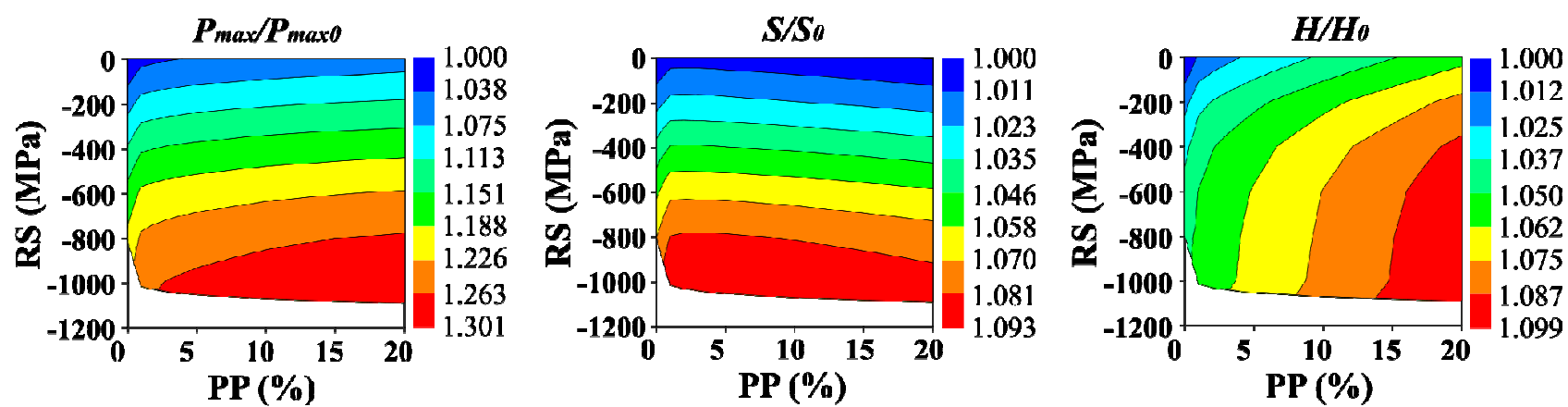

Fig. 2 Contour plots of normalized parameters obtained from FE simulation vs RS and PP. (The area in white corresponds to domain beyond- $\sigma_{y}$, which is impossible to reach elastically.)

Several features can be observed from these plots:

- The maximum load $P_{\max }$ is the most sensitive parameter because it varies by about $30 \%$ between the untreated material and the material with the highest RS and PP levels. Corresponding variations for the contact stiffness $S$ and the hardness $H$ are about $10 \%$.

- After $1 \%$ of plastic pre-strain, $P_{\max }$ and $S$ are mildly influenced by plasticity (PP) and mostly by residual stresses.

- The hardness is affected by both RS and PP. However, for residual stress levels above 600MPa (in absolute value), the hardness is almost unaffected by RS and varies with PP.

As the response, in terms of the two quantities RS and PP, of the 3 normalized parameters $P_{\max }, S$ and $H$ are significantly different, an inverse method can be proposed: the knowledge of two of the 3 indentation parameters, for instance $S$ and $H$, gives enough information to compute the residual stress and the accumulated plastic strain.

In order to illustrate the method from a purely numerical point of view, simulations of two combinations of RS and PP are performed. Combination $1\left(\mathrm{C}_{1}\right)$ is $\mathrm{PP}=11.5 \%$ and $\mathrm{RS}=-830 \mathrm{MPa}$, and combination $2\left(\mathrm{C}_{2}\right)$ is $\mathrm{PP}=16.5 \%$ and $\mathrm{RS}=-450 \mathrm{MPa}$. $\mathrm{C}_{1}$ is chosen in the region where hardness is almost independent of residual stress. Both $\mathrm{C}_{1}$ and $\mathrm{C}_{2}$ are taken in a range where $P_{\max }$ and $S$ exhibit a weak dependence to plastic strain. The values of $P_{\max } / P_{\max 0}, S / S_{0}$ and $H / H_{0}$ obtained numerically from these combinations are shown in Table 2.

Table 2 Values of parameters obtained from FE simulations of two sets of RS and PP

\begin{tabular}{cccccc}
\hline $\begin{array}{c}\text { Combinations of } \\
\mathrm{RS} \text { and PP }\end{array}$ & $\mathrm{PP}$ & $\mathrm{RS}$ & $P_{\max } / P_{\max 0}$ & $S / S_{0}$ & $H / H_{0}$ \\
\hline $\mathrm{C}_{1}$ & 11.5 & {$[\mathrm{MPa}]$} & - & - & - \\
$\mathrm{C}_{2}$ & 16.5 & -430 & 1.264 & 1.082 & 1.080 \\
& & & 1.189 & 1.047 & 1.085 \\
\hline
\end{tabular}

The values of RS and PP derived from the proposed method are listed in Table 3. It shows that for both $\mathrm{C}_{1}$ and $\mathrm{C}_{2}$, the derived values of RS and PP from the knowledge of the indentation quantities $\left(P_{\max } / P_{\max 0}, S / S_{0}\right.$ or $\left.H / H_{0}\right)$ are a little bit higher than the values imposed in the FEM model (less than or about 1\%). Fig. 3 shows graphically the solution for the 3 different couples of input values that can be used to determine PP and RS.

Table 3 RS and PP values derived from the inverse method.

\begin{tabular}{ccccccc}
\hline & \multicolumn{2}{c}{$\mathrm{C}_{1}(\mathrm{RS}=-830 \mathrm{MPa}, \mathrm{PP}=11.5 \%)$} & \multicolumn{2}{c}{$\mathrm{C}_{2}(\mathrm{RS}=-450 \mathrm{MPa}, \mathrm{PP}=16.5 \%)$} \\
\cline { 2 - 7 } & $P_{\max } / P_{\max 0}$ & $P_{\max } / P_{\max 0}$ & $S / S_{0}$ & $P_{\max } / P_{\max 0}$ & $P_{\max } / P_{\max 0}$ & $S / S_{0}$ \\
& $-S / S_{0}$ & $-H / H_{0}$ & $-H / H_{0}$ & $-S / S_{0}$ & $-H / H_{0}$ & $-H / H_{0}$ \\
\hline $\mathrm{RS}$ & $-837.9 \mathrm{MPa}$ & $-838.3 \mathrm{MPa}$ & $-837.6 \mathrm{MPa}$ & $-453.4 \mathrm{MPa}$ & $-453.1 \mathrm{MPa}$ & $-453.8 \mathrm{MPa}$ \\
$\mathrm{PP}$ & $11.62 \%$ & $11.61 \%$ & $11.61 \%$ & $16.51 \%$ & $16.60 \%$ & $16.59 \%$ \\
\hline
\end{tabular}




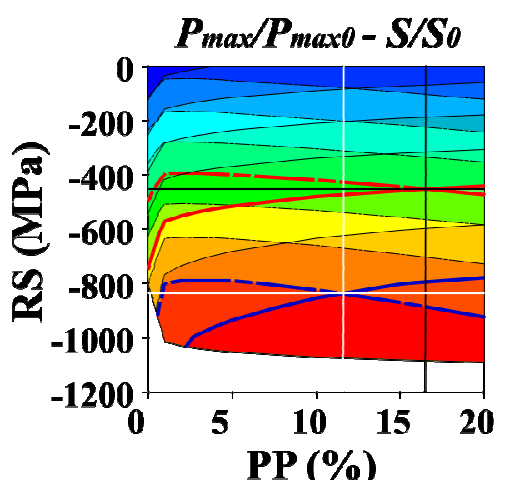

$\begin{array}{cc} & P \max / P \max 0 \\ \mathrm{C} 1 & \mathrm{~S} / \mathrm{S} 0 \\ & \mathrm{H} / \mathrm{Ho}\end{array}$
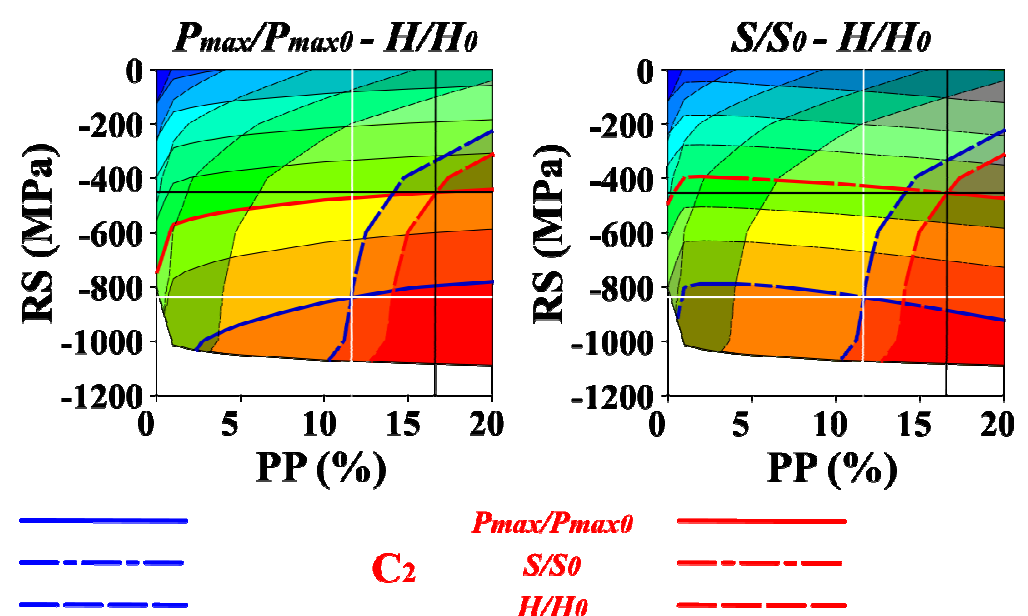

$\mathrm{C}_{2}$

Fig. 3 Identification of RS and PP values through the cross point of isolines of $C_{1}$ and $C_{2}$ on the pair-wise superposition of the contour plot of different parameters.

In order to investigate the stability of the method versus a noise on the input values, $a \pm 0.5 \%$ fluctuation was added into each indentation parameter $P_{\max } / P_{\max 0}, H / H_{0}$ and $S / S_{0}$ listed in Table 2 . The isolines of different pair of parameters are shown in Fig. 4. With different pair of parameters, the dispersion range on the RS value for $\mathrm{C}_{1}$ goes from $150 \mathrm{MPa}$ to $200 \mathrm{MPa}$, while for $\mathrm{C}_{2}$ it goes from $70 \mathrm{MPa}$ to $150 \mathrm{MPa}$. Concerning the $\mathrm{PP}$, the dispersion range for $\mathrm{C}_{1}$ goes from $5 \%$ to $10 \%$, while for $\mathrm{C}_{2}$ it goes from $5 \%$ to $15 \%$. For both $\mathrm{C}_{1}$ and $\mathrm{C}_{2}$, the smallest dispersion could be obtained when considering $P_{\max } / P_{\max 0}$ and $\mathrm{H} / \mathrm{H}_{0}$ as input values. On the contrary a strong dispersion can be observed when the pair $P_{\max }$ and $S$ is chosen. This can be explained by the fact that the isovalue lines cross with an acute angle which means that one of the two functions has a weak gradient versus one parameter (PP). When $S$ and $H$ are used, the isovalue lines cross with an angle close to $90^{\circ}$ but the gradients of the two functions are weak so the sensitivity is decreased.
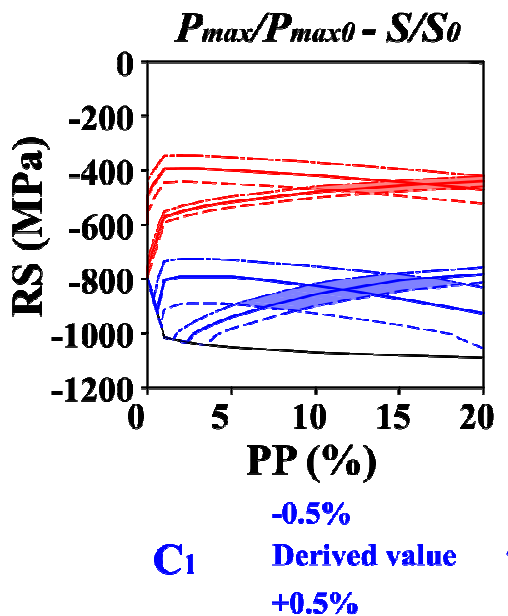

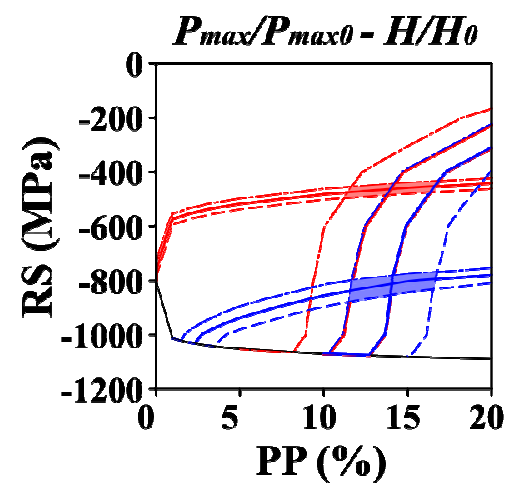

$\begin{array}{ll} & -0.5 \% \\ \mathrm{C}_{2} & \text { Derived value } \\ & +\mathbf{0 . 5 \%}\end{array}$
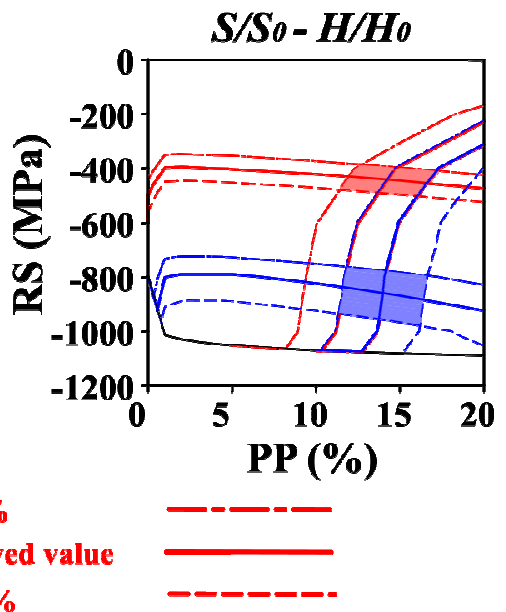

Fig. 4 Investigation of dispersion of the method proposed in this study.

These two examples show that the sensitivity of the method varies significantly with the region of the (PP-RS) space explored and with the pair of functions ( $P_{\max }$ and $S, P_{\max }$ and $H, S$ and $H$ ) chosen. This means that, according to the constitutive behavior of the material and the expected magnitude of the residual stress and plastic strain, the functions used in the inverse method must be carefully chosen.

In the presented study, an equi-biaxial residual stress state was investigated, but according to Giannakopoulos indentation is mostly sensitive to the hydrostatic component of the stress tensor [2], thus the results can be easily transposed to other stress states. Furthermore, the method presented here requires an accurate knowledge of the constitutive behavior of the initial material and does not take into account the effect of kinematic hardening. 


\section{Conclusion}

A method based on FE simulation was proposed for characterizing the residual stress state and the work hardening (plastic pre-strain) induced simultaneously by the shot peening process. Through a series of simulations of instrumented indentation, it was found that, although the loading indentation curves can be affected in the same way by the two quantities, the combination of 2 indentation parameters, obtained from experimental loading/unloading curves can be used to estimate them. These two parameters must be chosen among three: the normalized maximum load $\left(P_{\max } / P_{\max 0}\right)$, the normalized contact stiffness $\left(S / S_{0}\right)$ and the normalized contact hardness $\left(H / H_{0}\right)$. The normalization is performed with respect to the values obtained on the untreated material or far enough from the treated area.

Purely numerical test were performed and it was found that the smallest dispersion could be obtained for the pair $\left(P_{\max } / P_{\max 0}-H / H_{0}\right)$.

It was also observed that the sensitivity of the method decreases for high residual stress values, close to the compressive yield stress, and that this decrease affects the 3 parameters $P_{\max }, H$ and $S$.

In future studies, the method will be extended to spherical indenters and to more complex residual stress fields. The stability of the method can also probably be increased through a simultaneous use of the 3 parameters to determine residual stresses and work-hardening.

Experiments are necessary for this method. In the near future, instrumented indentation experiments with both Berkovich and spherical indenters will be performed on shot peened samples to test the practicability of this method. X-ray diffraction tests will be performed as well as comparison to check its reliability and precision. Measurements on specimens which have been submitted to tensile tests (no residual stress) or bending tests (with both residual stress and plastic strain) will also be performed.

Acknowledgement: Yu Gang Li would like to thank the Chinese Scholarship Council (CSC) for the financial support of his $\mathrm{PhD}$ in France.

\section{References}

[1] S. Carlsson, P.L. Larsson, On the determination of residual stress and strain fields by sharp indentation testing.: Part II: experimental investigation, Acta Materialia. 49 (2001) 2193-2203.

[2] A. Giannakopoulos, The influence of initial elastic surface stresses on instrumented sharp indentation, Journal of applied mechanics, 70 (2003) 638-643.

[3] J.I. Jang, Estimation of residual stress by instrumented indentation: A review, J. Ceram. Process. Res, 10 (2009) 391-400.

[4] S. Suresh, A. Giannakopoulos, A new method for estimating residual stresses by instrumented sharp indentation, Acta Materialia, 46 (1998) 5755-5767.

[5] T. Tsui, W. Oliver, G. Pharr, Influences of stress on the measurement of mechanical properties using nanoindentation: Part I. Experimental studies in an aluminum alloy, Journal of Materials Research, 11 (1996) 752-759.

[6] Y.G. Li, P. Kanouté, M. François, Influence of residual stress and work hardening on instrumented indentation, Matériaux \& Techniques, 101 (2013) 306.

[7] I.N. Sneddon, The relation between load and penetration in the axisymmetric Boussinesq problem for a punch of arbitrary profile, International Journal of Engineering Science, 3 (1965) 47-57.

[8] J.C. Hay, A. Bolshakov, G. Pharr, A critical examination of the fundamental relations used in the analysis of nanoindentation data, Journal of Materials Research, 14 (1999) 2296-2305. 